\title{
Herpes zoster infection following mRNA COVID-19 vaccine in a patient with ankylosing spondylitis
}

\author{
B. Maranini ${ }^{1}$, G. Ciancio ${ }^{1}$, R. Cultrera ${ }^{2}$, M. Govoni ${ }^{1}$ \\ ${ }^{1}$ Rheumatology Unit, Department of Medical Sciences, University of Ferrara; \\ ${ }^{2}$ Infectious Diseases, Department of Translational Medicine, University of Ferrara
}

\section{SUMMARY}

Since the severe acute respiratory syndrome-related coronavirus 2 (SARS-CoV-2) pandemic outbreak, vaccines gained a growing role. Possible vaccine-related side effects range from minor local events to more prominent systemic manifestations up to anaphylactic reactions.

A heterogeneous spectrum of cutaneous reactions has been reported, ranging from local injection site reactions to urticarial and morbilliform eruptions, pernio/chilblains and zoster flares.

Here, we describe a case of varicella zoster virus reactivation following mRNA coronavirus 2019 vaccine and discuss the available literature upon the topic published so far.

Key words: COVID-19, COVID-19 vaccine, herpes zoster, rheumatology.

Reumatismo, 2021; 73 (3): 174-176

Corresponding author: Beatrice Maranin Rheumatology Unit, Department of Medical Sciences, University of Ferrara
The current severe acute respiratory syndrome-related coronavirus 2 (SARS$\mathrm{CoV}-2$ ) pandemic is a worldwide challenge with strong medical and socioeconomic consequences. Enormous hopes were placed in the development of several vaccines, in the effort to relieve hospitalizations, severe disease and deaths related with coronavirus 2019 (COVID-19). The first clinical trials involved messenger RNA (mRNA) vaccines, which reportedly confer up to $95 \%$ protection from COVID-19 infection after a two-shot series (1). As the vaccination campaign is growing, surveillance of adverse effects needs to be monitored closely.

Possible side effects range from minor events such as sore arm and swelling at the injection site to more prominent manifestations such as fever and chills, headache, nausea, and anaphylactic reactions (1). $\mathrm{Cu}-$ taneous reactions after mRNA vaccines have been reported but are not well characterized.

Here, we describe a case of varicella zoster virus (VZV) reactivation occurring after mRNA vaccination.
A 41-year-old female patient, followed at our Rheumatology Unit because suffering from HLA-B27 related ankylosing spondylitis started five years earlier, came urgently to our clinic for the recent appearance of an itchy and tender skin rash over the volar surface of the right forearm, which occurred 7 days after the first dose of mRNA COVID-19 vaccine BNT162b2.

The patient had started immunosuppressive treatment with the TNF-inhibitor adalimumab three years earlier and since then the disease has been in persistent remission. The last dose of adalimumab was taken 15 days before receiving COVID-19 vaccination. Her main comorbidities were migraine and an annually monitored microprolactinoma. Her recent medical history did not include other relevant clinical symptoms such as fever, chills, dyspnea or cough.

Her GP found the skin lesions to be consistent with herpes zoster and a systemic antiviral treatment with acyclovir $800 \mathrm{mg}$ five times per day was started (2). When we first examined the patient, she was at day 7 


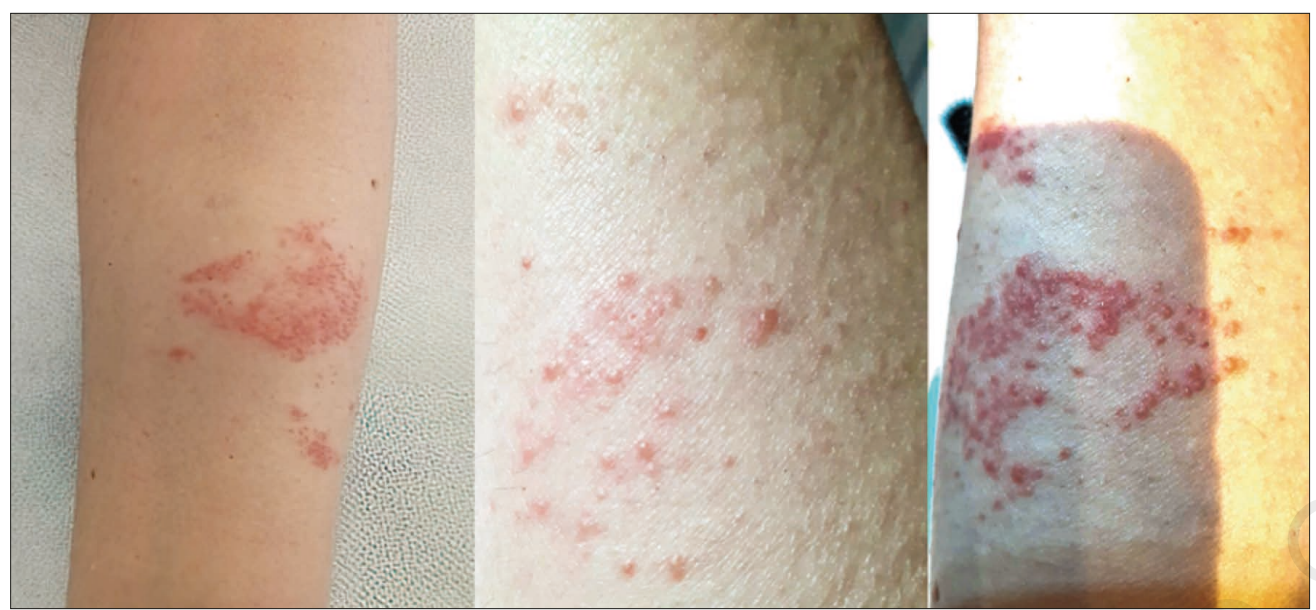

Figure 1 - Eruption of herpetic vesicles on the right forearm of the patient.

of the antiviral treatment and reported a progressive improvement of the lesion. On dermatological examination, a confluence of painful vesicles was appreciated over the right arm with a dermatomal distribution (Figure 1).

Based on clinical and serologic findings, the infectious disease consultant confirmed the diagnosis of herpes zoster infection and suggested to temporarily discontinue treatment with adalimumab and postpone the administration of the second vaccine dose for 6-7 weeks. Serological tests for the detection of anti-zoster antibodies showed high titer of $\operatorname{IgG}(1618 \mathrm{mUI} / \mathrm{mL}$, positive cut-off rate $>165$ ) with negative IgM antibodies. The polymerase chain reaction on vesicle fluid was not performed considering the high probability of negative analysis after seven days of antiviral therapy.

\section{DISCUSSION}

VZV is a neurotropic virus which, after primary infection, becomes latent in ganglia. Reactivation of VZV may occur spontaneously or can be triggered by trauma, stress, fever, immunosuppression, other infections and the administration of vaccines, which can lead to virus replication, inflammation and cell death in the tissues innervated by the involved neurons (3).

COVID-19 itself may represent a trigger for herpes zoster reactivation. Moreover, some cases of reactivation of VZV after
COVID-19 vaccine have been reported so far in literature (4). Eid et al. first reported herpes zoster reactivation following mRNA COVID-19 vaccine (3), while Bostan et al. first reported the resurfacing of herpes zoster after the employment of inactivated COVID-19 vaccine (5).

In a study conducted by McMahon et al. (6), morphology and timing of cutaneous reactions after mRNA COVID-19 vaccines were evaluated through a provider-facing registry-based study. From December 2020 to February 2021, 414 cutaneous reactions to mRNA COVID-19 vaccines from Moderna $(83 \%)$ and Pfizer (17\%) were recorded. Delayed large local reactions were most common, followed by local injection site reactions, urticarial eruptions, and morbilliform eruptions. Other less common cutaneous findings included pernio/chilblains, zoster, herpes simplex flares and pityriasis rosea-like reactions.

In their observational study, Furer et al. (7) reported some cases of herpes zoster reactivation following the BNT162b2 mRNA COVID-19 vaccination in patients with autoimmune inflammatory rheumatic diseases (AIIRD). The study was conducted in two Rheumatology Departments in Israeli patients with AIIRD $(n=491)$ and controls $(n=99)$. The prevalence of herpes zoster reactivation among rheumatological patients was $1.2 \%$ (6 patients) versus none in controls. The patients were women aged $49 \pm 11$ years; four were affected by rheumatoid 
arthritis (RA), one by Sjogren's syndrome, and another one by undifferentiated connective tissue disease. Five patients developed the first in a lifetime event of VZV within a short time after the first vaccine dose and the remaining one after the second vaccine dose.

In most cases, VZV infection was mild, except for a case of herpes zoster ophtalmicus, without corneal involvement, in a RA patient treated with tofacitinib. Five patients received antiviral therapy with a complete resolution of symptoms within 6 weeks. None of the patients developed disseminated disease or post-herpetic neuralgia. Notably, one patient developed VZV reactivation despite being vaccinated for herpes zoster two years prior to the reported case. Five patients completed the second vaccine dose without any other adverse effects.

Although the underlying causes of VZV reactivation following mRNA COVIDQ19 vaccinations have not been clearly established yet, potential mechanisms might be related to stimulation of innate immunity through toll-like receptors by mRNA-based vaccines (8).

The main goal of this report is to further raise awareness about the possible reactivation of VZV in patients with autoimmune diseases who have a higher risk of herpes zoster reactivation compared to the healthy population (9). Indeed, the safety profile of COVID-19 vaccines among patients with rheumatic diseases has not been tested during vaccine development, because patients treated with immunosuppressive agents have been excluded from initial clinical trials.

The close temporal occurrence of VZV reactivation after COVID-19 vaccination, as well as the cases from the literature, support the question of a potential link between these events, even though it is not possible to establish a straightforward relationship between them.

What is relevant to understand is the incidence of VZV reactivation following SARS-
CoV-2 vaccines, when compared with other viral vaccines, particularly considering that VZV is a cause of considerable morbidity, especially in elderly and critically ill patients. Further elucidation will derive from the ongoing global scale vaccine campaign.

\section{Conflict of interest}

None.

\section{Funding source}

None.

\section{REFERENCES}

1. Polack FP, Thomas SJ, Kitchin N, et al. Safety and efficacy of the BNT162b2 mRNA COVID-19 vaccine. N Engl J Med. 2020; 383 (27). [Epub ahead of print].

2. Cohen JI. Clinical practice: Herpes zoster. N Engl J Med. 2013; 369: 255-63.

3. Walter R, Hartmann K, Fleisch F, et al. Reactivation of herpesvirus infections after vaccinations? Lancet (London, England). 1999; 353: 810 .

4. Bostan E, Yalici-Armagan B. Herpes zoster following inactivated COVID-19 vaccine: a coexistence or coincidence? J Cosmet Dermatol. 2021; 20: 1566-7.

5. Eid E, Abdullah L, Kurban M, Abbas O. Herpes zoster emergence following mRNA COVID-19 vaccine. J Med Virol. 2021; 93: 5231-2.

6. McMahon DE, Amerson A, Rosenbach M, et al. Cutaneous reactions reported after Moderna and Pfizer COVID-19 vaccination: a registry-based study of 414 cases. J Am Acad Dermatol. 2021; 85: 46-55.

7. Furer V, Zisman D, Kibari A, et al. Herpes zoster following BNT162b2 mRNA COVID-19 vaccination in patients with autoimmune inflammatory rheumatic diseases: a case series. Rheumatology (Oxford, England). 2021. [Epub ahead of print].

8. Zhang C, Maruggi G, Shan H, Li J. Advances in mRNA vaccines for infectious diseases. Front Immunol. 2019; 10: 594.

9. Furer V, Rondaan C, Heijstek M, et al. Incidence and prevalence of vaccine preventable infections in adult patients with autoimmune inflammatory rheumatic diseases (AIIRD): a systemic literature review informing the 2019 update of the EULAR recommendations for vaccination in adult patients with AIIRD. RMD Open. 2019; 5: e001041. 